\title{
Parity Associated with Long-Term Disease Progression in Women with Multiple Sclerosis
}

Barbara E. Teter ${ }^{1,2,3}$, Katelyn S Kavak ${ }^{1,2}$, Channa Kolb- Sobieraj ${ }^{1,2,3}$, Patricia K Coyle K $^{2,3}$ and Bianca Weinstock-Guttman ${ }^{1,2,3^{*}}$ on behalf of the New York State multiple sclerosis consortium

${ }^{1}$ Jacobs Comprehensive MS Treatment and Research Center, University at Buffalo, Buffalo, NY, USA

${ }^{2}$ New York State Multiple Sclerosis Consortium, University at Buffalo, Buffalo, NY, USA

${ }^{3}$ Neurology, SUNY at Stony Brook, Stony Brook, NY, USA

"Corresponding author: Bianca Weinstock-Guttman MD, New York State multiple sclerosis Consortium Research Office, Buffalo, NY, USA, Tel: 716-859-7051; Fax: 716-859-7573; E-mail: bweinstock-guttman@kaleidahealth.org

Received date: Mar 18, 2014, Accepted date: Apr 24, 2014, Published date: Apr 26, 2014

Copyright: ( 2014 Barbara T, et al. This is an open-access article distributed under the terms of the Creative Commons Attribution License, which permits unrestricted use, distribution, and reproduction in any medium, provided the original author and source are credited.

\section{Abstract}

Background: Pregnancy in multiple sclerosis (MS) is marked by a decrease in relapse activity with a corresponding rebound in the first months postpartum. Because MS typically occurs during childbearing years, it is important to determine the long term effect of pregnancy.

Design/Methods: Subjects were part of the New York State Multiple Sclerosis Consortium registry. 1,195 parous and 328 nulliparous women with clinically definite MS age 45 or older were analyzed to determine time to a disability milestone, Expanded Disability Status Scale (EDSS) 6.0. A Cox proportional hazards model was used to examine the effect of parity on time from MS disease onset to EDSS 6.0, adjusted for confounding factors.

Results: The average disease duration for 1,523 women was 18.1 years. During a mean follow-up period of 5.6 years $23.1 \%$ of the parous group reached EDSS 6.0 , compared to $26.5 \%$ of the nulliparous women. Cox survival curves between parous and nulliparous women were significantly different $(\mathrm{HR}=.68, \mathrm{Cl}=.53-.87, \mathrm{p}=.002)$, with parous women showing a longer time to reach the disability outcome. Follow-up analyses stratified by MS type showed that parity remained a significant predictor of disease progression in relapsing remitting (RRMS) patients, whereas this effect was not observed within progressive subtypes.

Conclusions: Our results demonstrate that parous women with RRMS take a longer time to reach a well-defined disability milestone (i.e. use of cane) compared to nulliparous women, suggesting that pregnancy may convey a long term benefit. Further research is needed to determine why pregnancy might be protective against long term MS disability progression.

Keywords: Multiple sclerosis; Pregnancy; Prognosis; Disease progression; Women; Reproductive

\section{Introduction}

Similar to other immune mediated diseases $[1,2]$, pregnancy was shown to influence Multiple Sclerosis (MS) disease activity. It is marked by a decrease in relapse rate, especially during the third trimester. A temporary increase in relapses is observed within the first months postpartum, after which the rate returns to pre-pregnancy baseline [3-5]. A recent case-control study found an association between both pregnancy and a higher number of offspring with a decreased risk of a first demyelinating event [6]. The protective effects of pregnancy on MS disease activity are thought in part to reflect the anti-inflammatory effects of sex hormones. Sex hormones such as estrogen and progesterone are increased during pregnancy, with the highest levels reported in the third trimester, and a sharp drop postpartum [7]. There is a sex hormone mediated shift in immune response during pregnancy [8], which is characterized by a decrease in pro-inflammatory $\mathrm{TH} 1$ responses and an increase in antiinflammatory $\mathrm{TH} 2$ responses, intended to promote fetal survival [9-11].
When investigating long term effects of pregnancy on disease progression, results are conflicting. Some studies report a long term beneficial effect on MS disease course among women who had children compared to those who did not [12-15], but other studies do not $[5,16-18]$.

Because MS most often occurs during childbearing years and many couples are concerned about the consequences of childbirth, it is important to determine whether pregnancy has long term effects on disease course. We extracted a large sample of MS patients to investigate the effects of pregnancy on disability progression.

\section{Methods}

All subjects were part of the New York State Multiple Sclerosis Consortium (NYSMSC) registry. The NYSMSC was founded in 1996 and is one of the largest ongoing databases of MS patients, as described previously [19]. Data for this study was collected from 13 academic centers. The strengths of the dataset are based on its longitudinal prospective characteristics and availability of demographic and physician reported clinical measurements and MS treatment as well as patient reported disability outcomes and psychosocial function. 
Informed consent was obtained from all patients for inclusion in the study in compliance with Institutional review board (IRB) policies for research with human subjects. The present retrospective cohort study was based on longitudinal data (1997-2012) extracted from the NYSMSC database $(n=9,993)$. Only women who were 45 years or older at the time of the last available follow- up visit, with clinically definite MS were included leaving us a sample size of 3,872. Comparison of the NYSMSC sample to other large databases in the United States (US) shows our data represents the general MS population in the US. We chose a score of 6.0 on the Kurtzke Expanded Disability Status Scale (EDSS), indicating "Intermittent or unilateral constant assistance (cane, crutch or brace) required to walk 100 meters with or without resting" [20], as a known disability outcome. Parity was assessed longitudinally and subjects were subsequently divided in the parous (women having borne at least one child) or nulliparous group (women who have not borne a child). Exclusion criteria consisted of subjects with missing data on key variables, had no follow-up data, and those who reached EDSS 6.0 at or before registration leaving a total sample size of 1,523 (Figure 1). The mean time between registration and first follow-up and years between subsequent follow-ups was 0.99 years (SD 1.11).

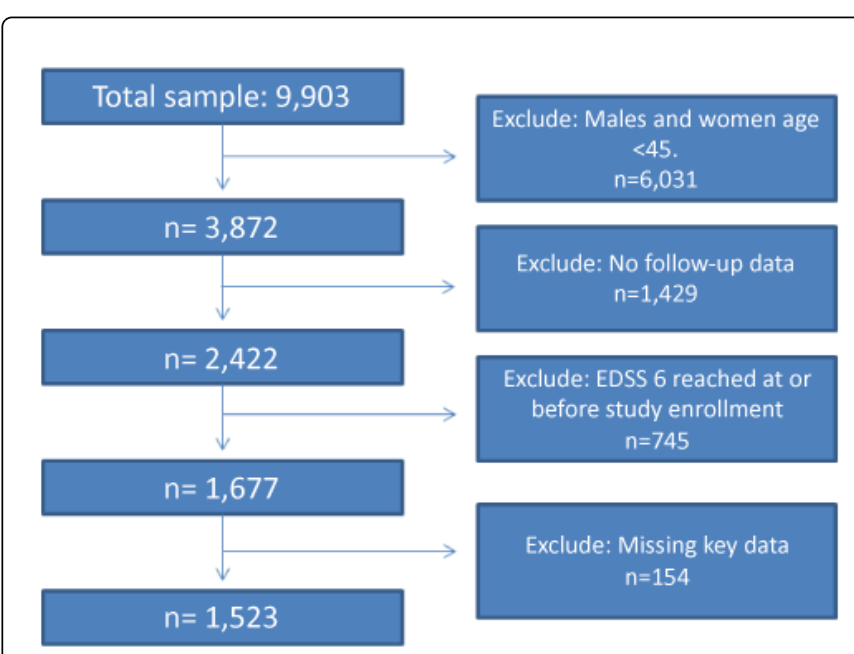

Figure 1: Flow chart showing patient exclusions

To analyze time to disability, a time to event variable was created using the time between MS symptom onset and the follow up date where EDSS 6.0 was first reached. Time between MS symptom onset and the date of most recent follow-up was used for subjects who did not reach EDSS 6.0.

Demographic and disease characteristics were compared between the parous and nulliparous groups, using Student's t-tests and Chisquare tests. Square root and logarithmic transformations were carried out to normalize non-normally distributed variables. A MannWhitney $\mathrm{U}$ test was used to compare groups when transformations did not yield improved data distributions.

Kaplan-Meier survival curves were calculated to examine time from MS disease onset to the disability milestone of EDSS 6.0. Log-rank statistics were used to assess differences between parity groups. This was followed by Cox proportional hazard regression models, which allowed for adjustment of the confounding factors age at symptom onset, type of MS disease at registration, education and disease modifying therapy (DMT) use (either before, at, or after study enrollment). To assess for multicollinearity, the variable inflation factor (VIF) for each predictor was determined. Furthermore, the proportional hazard assumption was checked using a log-minus-log plot. We considered a two-sided p-value of $<.05$ as statistically significant. Data was analyzed using Statistical Package for Social Sciences (SPSS) for Windows (version 21, SPSS Inc., Chicago, IL, USA).

\section{Results}

Table 1 shows the general characteristics of this study population. Our analysis included 1,195 (78.5\%) parous and 328 (21.5\%) nulliparous women. Mean age at onset of MS symptoms was 36.2 years, age at MS diagnosis was 41.6 years and mean age at most recent visit was 54.3 years (range: 45-82). The mean follow-up time of our sample was 5.6 years $(\mathrm{SD}=3.7)$, with an average disease duration of 18.1 years $(\mathrm{SD}=9.6)$.

\begin{tabular}{|c|c|c|c|c|}
\hline & $\begin{array}{l}\text { Total } \\
(n=1523)\end{array}$ & $\begin{array}{l}\text { Parous } \\
(n=1195)\end{array}$ & $\begin{array}{l}\text { Nulliparous } \\
(n=328)\end{array}$ & $p$-value \\
\hline Age at MRV (SD) & $54.3(6.9)$ & $54.6(7.1)$ & $53.3(5.9)$ & .015 \\
\hline $\begin{array}{l}\text { Age at MS onset } \\
\text { (SD) }\end{array}$ & $36.2(9.6)$ & $36.3(9.9)$ & $35.6(8.7)$ & NS \\
\hline $\begin{array}{lr}\begin{array}{lr}\text { MS } \\
\text { duration }\end{array} & \text { disease } \\
\text { (SD) } & \text { (years) }\end{array}$ & $18.1(9.6)$ & $18.2(9.9)$ & $17.7(8.5)$ & NS \\
\hline Race, N (\%) & & & & NS \\
\hline Caucasian & $1433(94.2)$ & 1127 (94.5) & 306 (93.6) & \\
\hline African-American & $71(4.7)$ & $53(4.4)$ & $18(5.5)$ & \\
\hline Other & $16(1.1)$ & $13(1.1)$ & $3(0.9)$ & \\
\hline Education, $\mathbf{N}(\%)$ & & & & $<.001$ \\
\hline$\leq$ High school & $484(32.1)$ & 407 (34.3) & $77(23.8)$ & \\
\hline Some college & $416(27.5)$ & $342(28.8)$ & 74 (22.9) & \\
\hline College/university & 341 (22.6) & $252(21.2)$ & $89(27.6)$ & \\
\hline Post-graduate & $269(17.8)$ & $186(15.7)$ & $83(25.7)$ & \\
\hline DMT use, $\mathbf{N}(\%)$ & $1271(83.5)$ & $995(83.3)$ & $276(84.1)$ & NS \\
\hline $\begin{array}{l}\text { Type of MS at } \\
\text { registration, } \mathrm{N}(\%)\end{array}$ & & & & NS \\
\hline RR & $1206(79.3)$ & 936 (78.5) & $270(82.3)$ & \\
\hline SP & $216(14.2)$ & $179(15.0)$ & $37(11.3)$ & \\
\hline PR/PP & $99(6.5)$ & $78(6.5)$ & $21(6.4)$ & \\
\hline EDSS at MRV (SD) & $3.5(1.9)$ & $3.5(1.9)$ & $3.6(2.0)$ & NS \\
\hline EDSS $\geq 6.0 \mathrm{~N},(\%)$ & $363(23.8)$ & $276(23.1)$ & 87 (26.5) & NS \\
\hline
\end{tabular}

Table 1: Demographic and clinical characteristics.

MRV: Most Recent Visit; DMT: Disease Modifying Therapy; RR: Relapsing Remitting; SP: Secondary Progressive; PR: Primary Relapsing; PP: Primary Progressive; EDSS: Expanded Disability Status Scale 
Page 3 of 6

The predominant clinical subtype of MS at registration was Relapsing-Remitting (RR) MS (79.3\%), followed by Secondary Progressive (SP) MS (14.2\%), and Primary Progressive or Progressive Relapsing (PP-PR) MS (6.5\%), whereas at most recent follow-up we observed $66.8 \%, 27.5 \%$ and $6.5 \%$ respectively. During the course of this study, $363(23.8 \%)$ subjects reached EDSS 6.0, parous women taking 220.3 months and nulliparous women taking 215.6 months. Furthermore, 1,271 (83.5\%) of our sample reported ever using DMT. The only significant difference in demographic factors involved education and age at most recent visit; nulliparous women had a higher level of education than parous women $(\mathrm{p}<.001)$ and were slightly younger $(\mathrm{p}=.015)$ (Table 1$)$.

Kaplan Meier curves were calculated to determine the unadjusted relationship between parity and disease progression. A log rank test indicated that parous women reached EDSS 6.0 later (median $=425$ months, $\mathrm{CI}=382-467$ months) than nulliparous women (median=369 months, $\mathrm{CI}=305-433$ months, $\mathrm{p}=.032$ ).

Low VIF values $(<2.5)$ were observed between the independent variables, indicating there was no interfering level of multicollinearity. The proportional hazard assumption was not violated as determined by log-minus-log plots. Cox proportional hazards analysis allowed for investigating the effect of parity on the risk of reaching EDSS 6.0, while adjusting for confounding factors. Survival curves between parous and nulliparous women were significantly different $(\mathrm{HR}=.69, \mathrm{CI}=.54-.89$, $\mathrm{p}=.004$ ), with parous women showing longer time to disability (Figure 2). Education levels did not significantly contribute to the model. Time-to-event analysis yielded significant differences between RRMS and progressive MS types ( $\mathrm{HR}=.63, \mathrm{CI}=.44-.91, \mathrm{p}=.013)$. Because of observed significant group-wise differences, additional follow-up analyses stratified by MS type showed parity remained a significant predictor of disease progression in RRMS patients $(\mathrm{p}=.026)$, whereas within progressive subtypes this effect was not observed.

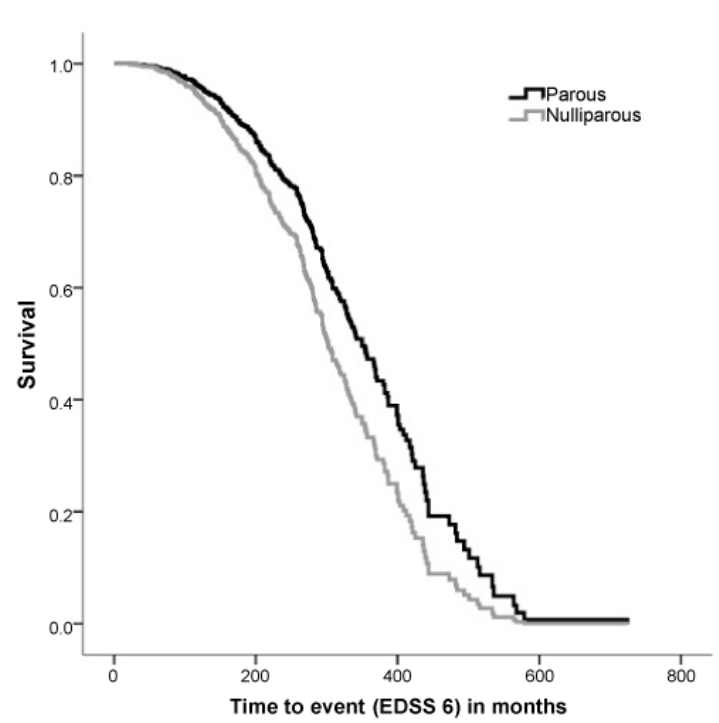

Figure 2: Cox survival curves of parous and nulliparous women to reach the disability milestone.

\section{Discussion}

This study found that parous MS women take a longer time to reach EDSS 6.0, a well-accepted MS disability milestone, compared to nulliparous women. This is consistent with pregnancy conveying a favorable long term prognosis in MS, an important finding considering most demographic characteristics did not differ between parous and nulliparous groups. The statistical analyses remained significant after adjusting for the effects of confounding variables. Although there were group wise differences in education level, this was not significantly related to EDSS progression. Analyses stratified by MS type indicated that parity was a significant predictor of disability progression only within the RRMS cohort. This suggests that pregnancy may have a different impact during the progressive phase of the disease. These results are in line with D'hooge et al. [13] who also show an effect of parity in RRMS but not in the progressive onset types of MS. Previous studies have shown that pregnancy is beneficial for the short term in MS. The well-known Pregnancy and Multiple Sclerosis (PRIMS) trial reported a significant reduction of relapses during pregnancy (70\% in the last trimester), with a sharp rebound to $70 \%$ above pre-pregnancy relapse rates observed during the first three months postpartum. Our data is consistent with Runmarker et al. [15] who were one of the first to report a long term benefit of parity on MS disease course. Positive long term effects of parity have also been reported by others, using EDSS 6.0 as the outcome measure $[14,13]$. However, other studies showed conflicting results, but follow-up periods tended to be inconsistent or too short (3 to 12 months postpartum) [21,22]. In a small 3-year prospective study, Worthington et al. [23] reported no significant differences between relapse rates, severity of relapses or MS disability at 3 years postpartum. Roullet et al. [24] followed 32 women with full-term pregnancies for a mean of 10.3 years, and concluded pregnancy did not lead to increased disability. Contrary to our study, Koch and colleagues [16] found that parity had no influence on the risk of secondary progression; however, this study did not examine disability status as a factor of disease progression and their sample size was much smaller compared to ours.

There are many immune and hormonal changes that can occur during pregnancy. The drop in relapse rates during pregnancy, and the increase in relapse rates seen shortly postpartum, coincide with the rise and fall of sex steroids including estrogen. Estrogen levels are highest during late pregnancy and dramatically decrease after delivery [7]. Estrogen may have an impact outside of pregnancy. The decline in estrogens observed in menopause is less researched, but one study reported that symptoms worsened in menopause but improved in women using hormone replacement therapy [25]. In an animal model of MS, experimental autoimmune encephalomyelitis (EAE), treatment with estriol led to a significant reduction in disease severity in both male [26] and female mice [26,27]. Another study researching the role of pregnancy hormones in MS found that low-dose preimplantation factor (PIF) administration in EAE mice led to complete reversal of MS-induced paralysis [28]. Studies using hormones in human subjects with MS are limited, but Sicotte et al. [29] found that non-pregnant women treated with oral estrogens showed reduction in number and volume of gadolinium enhancing lesions [29]. Clinical trials using sex steroids are currently underway [30,31]. Ponsonby et al. [6] investigated subjects at their first demyelinating event, and found a reduced risk of developing definite MS as the number of pregnancies increased. Other studies have found a decreased risk of developing MS with having children, observed in both in females and males, arguing for a possible behavioral, social protective impact of parenting 
influence on the immune system and indirectly on the risk for developing MS [32-34].

Autoimmune diseases are more prevalent in women compared to men, an effect that is likely influenced by sex hormones such as estrogens. The exact role estrogens play in ameliorating MS disease course is not fully understood. Estrogens have both immunological and neurological effects. MS shows an imbalance in favor of Th1 lymphocytes that promote the secretion of pro-inflammatory cytokines such as IFNy, IL2 and TNFa. During pregnancy the fetus has to be protected against the mother's immune response, and there is a corresponding immune shift towards anti-inflammatory Th2 lymphocytes and corresponding cytokine production such as IL-4, IL-10 and TGF $\beta$. The increased risk in relapses postpartum may be due to the maternal shift back towards a Th1 milieu [35]. Similar beneficial outcomes of pregnancy have also been reported in other Th1 mediated diseases such as rheumatoid arthritis [36]. On the other hand the effects of a rise in estrogens can be deleterious on B-cell driven diseases such as systemic lupus erythematosus (SLE) in which increased frequency of diseases flares are observed during pregnancy [37]. Sex steroids are likely to mediate these immune shifts, as high levels of estrogens as seen during pregnancy promote antiinflammatory cytokines in T-cell clones obtained from patients with MS [38], while progesterone has been shown to decrease the level of Th1 cytokines such as IFN $\gamma$ and to induce the production of the Th2 cytokine IL-4.

Nonetheless the long term beneficial effect of pregnancy on MS disease progression as seen in our study is still difficult to fully understand. As most of the patients evaluated in this study were of menopausal age, a better ascertaining of the endocrine-immune system interactions and their relation to autoimmune diseases is of evident importance. Interestingly, estrogens after menopause are mainly synthesized and metabolized in peripheral tissues from androgens (usually known to have a protective anti-inflammatory effect) under the action of the aromatase complex [39]. Presence of inflammatory cytokines are usually enhancers of the aromatase activity that can be translated into an accelerated conversion of androgens towards estrogen metabolites. Environmental influences such as 1,25dihydroxyvitamin D3 were shown to have beneficial effects by down regulating aromatase expression and inflammatory cytokines [40].

An important confounding factor in pregnancy and MS research is the probable bias of women with severe disease symptoms bearing fewer children due to reduced reproductive activity or the fear of not being able to take care of a child [41], while women with less severe disease course might choose to become pregnant. In a previous analysis we observed an inverse trend between disability (EDSS) and number of births (0-6). The mean age of patients age 45 or higher (no child birth after age 40) at the point of most recent evaluation was 54.0 (SD 7.4) with nulliparous women having a higher EDSS mean of 4.73 (SD 2.4), parous women with 1 to 2 child-births having an EDSS mean 4.60 (2.3) and parous women with 3 to 6 child-births having an EDSS mean 4.55 (2.3). The trend was strongest in women age 50-53. A recent study found that concerns that MS symptoms would interfere with parenting was the most common reason for subjects with MS not to have children after being diagnosed [42]. We are in the process of researching the reasons why MS patients did not bear children.

A limitation of our study is that we lacked specific data for the temporality of onset and pregnancy. A large retrospective study examined time to EDSS 6.0 between women who did not have children, those who had children before MS onset, those who had children after MS onset and women who had children both before and after MS onset, and found that the difference in time to EDSS 6.0 was entirely accounted for by age at MS onset [43]. While we did not observe any differences in age at MS onset between the parous and nulliparous group, we did control for its effects in our Cox regression model because of the well-known association with our outcome variable. Results remained significant. Furthermore, we did not have data available to examine the number of pregnancies in relation to disability, an important confounder as others have found effects of number of pregnancies on disability outcome [6] and the risk of developing MS [32].

We are conducting further data collection for an in depth study to ascertain disability over time pointedly related to the temporal relationship between reproductive events and MS symptom onset and/or diagnosis as well as pre and post-partum DMT use, reasons for pregnancy planning and comprehensive breast-feeding history. Another limitation may be related to the recording of time to reach EDSS 6.0. In this study, measurements of EDSS were captured at (scheduled) follow-ups, and the exact date of reaching the disability milestone may be slightly different.

To summarize, this study supports the postulation that pregnancy has a favorable prognosis on long term disability in RRMS patients although causality cannot be assumed. Further studies are needed to clarify whether this is not true for progressive MS, and to determine the precise factors that explain pregnancy's long term benefit.

\section{Acknowledgements}

We would like to thank Stephanie Wasch-Redden for contributing to the manuscript, Karen Zakalik for supervising personnel and Kelly McGowan-Trzyzewski for editing the manuscript.

The authors acknowledge members of the NYSMSC: Keith Edwards (Empire Neurology, PC, Latham, NY), Andrew Goodman (University of Rochester Medical Center, Rochester, NY), Malcolm Gottesman (Winthrop Comprehensive MS Care Center, Mineola, NY), Joseph Herbert (NYU Langone Medical Center, New York, NY), Burk Jubelt (SUNY Upstate Medical University, Syracuse, NY), Ilya Kister (NYU Langone Medical Center, New York, NY), Lauren Krupp (SUNY Stony Brook, Stony Brook, NY), Michael Lenihan (Adirondack Neurology Associates, PC, Albany, NY), Fred Lublin (Mount Sinai School of Medicine, New York, NY), Aaron Miller (Mount Sinai School of Medicine, New York, NY), Krupa Pandey (Albany Medical College, Albany, NY), Allan Perel (Alpha Neurology, PC, Staten Island, NY), Carl Granger, (UDSMR, SUNY Buffalo, Buffalo NY) and Robert Zivadinov (BNAC, SUNY Buffalo, Buffalo, NY).

\section{Disclosures}

Barbara E. Teter has received grant and or research support from Biogen Idec, Teva Neurosciences, EMD Serono, Avanir, Genzyme and Novartis; Katelyn S. Kavak has nothing to disclose; Karen Zakalik has nothing to disclose; Dr.Channa Kolb- Sobieraj has served on the scientific advisory board for Teva and has received honoraria as a speaker for Teva, Biogen and EMD Serono; Patricia Coyle is a consultant for Acorda, Accordant, Bayer, Biogen Idec, Genzyme/ Sanofi, Genentech/Roche, Merck Serono, and Teva. This includes Advisory Boards for Acorda and Genzyme/Sanofi. Dr Coyle has spoken for and received travel funds from Genzyme/Sanofi. Dr Coyle has received research support from Actelion, Novartis and Opexa; Bianca Weinstock-Guttman received personal compensation for 
consulting, speaking and serving on a scientific advisory board for Biogen Idec, Teva Neuroscience, EMD Serono, Novartis, Questcor and Genzyme \& Sanofi. She received financial support for research activities from NMSS, NIH (not for the present study), ITN, Teva Neuroscience, Biogen Idec, EMD Serono, Aspreva, Novartis, Genzyme.

\section{Ethical Standards}

Informed consent was obtained from all patients for inclusion in the study in compliance with IRB policies in accordance with ethical standards for research in human subjects.

\section{Conflict of Interest}

This study was not sponsored and contributing authors have no conflicts of interest.

\section{References}

1. Raychaudhuri SP, Navare T, Gross J, Raychaudhuri SK (2003) Clinical course of psoriasis during pregnancy. Int J Dermatol 42: 518-520.

2. Nelson JL, Ostensen M (1997) Pregnancy and rheumatoid arthritis. Rheum Dis Clin North Am 23: 195-212.

3. Korn-Lubetzki I, Kahana E, Cooper G, Abramsky O (1984) Activity of multiple sclerosis during pregnancy and puerperium. Ann Neurol 16: 229-231.

4. Confavreux C, Hutchinson M, Hours MM, Cortinovis-Tourniaire P, Moreau T (1998) Rate of pregnancy-related relapse in multiple sclerosis. Pregnancy in Multiple Sclerosis Group. N Engl J Med 339: 285-291.

5. Vukusic S, Hutchinson M, Hours M, Moreau T, Cortinovis-Tourniaire P, et al. (2004) Pregnancy and multiple sclerosis (the PRIMS study): clinical predictors of post-partum relapse. Brain 127: 1353-1360.

6. Ponsonby AL, Lucas RM, van der Mei IA, Dear K, Valery PC, et al. (2012) Offspring number, pregnancy, and risk of a first clinical demyelinating event: the AusImmune Study. Neurology 78: 867-874.

7. Robinson DP, Klein SL (2012) Pregnancy and pregnancy-associated hormones alter immune responses and disease pathogenesis. Horm Behav 62: 263-271.

8. Whitacre CC, Reingold SC, O'Looney PA (1999) A gender gap in autoimmunity. Science 283: 1277-1278.

9. Liu F, Guo J, Tian T, Wang H, Dong F, et al. (2011) Placental trophoblasts shifted Th1/Th2 balance toward Th2 and inhibited Th17 immunity at fetomaternal interface. APMIS 119: 597-604.

10. Romagnani S (2006) Regulation of the T cell response. Clin Exp Allergy 36: 1357-1366.

11. Krishnan L, Guilbert LJ, Wegmann TG, Belosevic M, Mosmann TR (1996) $\mathrm{T}$ helper 1 response against Leishmania major in pregnant C57BL/6 mice increases implantation failure and fetal resorptions. Correlation with increased IFN-gamma and TNF and reduced IL-10 production by placental cells. J Immunol 156: 653-662.

12. Verdru P, Theys P, D'Hooghe MB, Carton H (1994) Pregnancy and multiple sclerosis: the influence on long term disability. Clin Neurol Neurosurg 96: 38-41.

13. D'hooghe MB, Haentjens P, Nagels G, D'Hooghe T, De Keyser J (2012) Menarche, oral contraceptives, pregnancy and progression of disability in relapsing onset and progressive onset multiple sclerosis. J Neurol 259: 855-861.

14. D'hooghe MB, Nagels G, Uitdehaag BM (2010) Long-term effects of childbirth in MS. J Neurol Neurosurg Psychiatry 81: 38-41.

15. Runmarker B, Andersen O (1995) Pregnancy is associated with a lower risk of onset and a better prognosis in multiple sclerosis. Brain 118 : 253-261.
16. Koch M, Uyttenboogaart M, Heersema D, Steen C, De Keyser J (2009) Parity and secondary progression in multiple sclerosis. J Neurol Neurosurg Psychiatry 80: 676-678.

17. Stenager E, Stenager EN, Jensen K (1994) Effect of pregnancy on the prognosis for multiple sclerosis. A 5-year follow up investigation. Acta Neurol Scand 90: 305-308.

18. Thompson DS, Nelson LM, Burns A, Burks JS, Franklin GM (1986) The effects of pregnancy in multiple sclerosis: a retrospective study. Neurology 36: 1097-1099.

19. Jacobs LD, Wende KE, Brownscheidle CM, Apatoff B, Coyle PK, et al. (1999) A profile of multiple sclerosis: the New York State Multiple Sclerosis Consortium. Mult Scler 5: 369-376.

20. Kurtzke JF (1983) Rating neurologic impairment in multiple sclerosis: an expanded disability status scale (EDSS). Neurology 33: 1444-1452.

21. Sadovnick AD, Eisen K, Hashimoto SA, Farquhar R, Yee IM, et al. (1994) Pregnancy and multiple sclerosis. A prospective study. Arch Neurol 51: 1120-1124.

22. Dwosh E, Guimond C, Duquette P, Sadovnick AD (2003) The interaction of MS and pregnancy: a critical review. Int MS J 10: 38-42.

23. Worthington J, Jones R, Crawford M, Forti A (1994) Pregnancy and multiple sclerosis--a 3-year prospective study. J Neurol 241: 228-233.

24. Roullet E, Verdier-Taillefer MH, Amarenco P, Gharbi G, Alperovitch A, et al. (1993) Pregnancy and multiple sclerosis: a longitudinal study of 125 remittent patients. J Neurol Neurosurg Psychiatry 56: 1062-1065.

25. Smith R, Studd JW (1992) A pilot study of the effect upon multiple sclerosis of the menopause, hormone replacement therapy and the menstrual cycle. Journal of the Royal Society of Medicine 85: 612-613.

26. Palaszynski KM, Liu H, Loo KK, Voskuhl RR (2004) Estriol treatment ameliorates disease in males with experimental autoimmune encephalomyelitis: implications for multiple sclerosis. Journal of neuroimmunology 149: 84-89.

27. Kim S, Liva SM, Dalal MA, Verity MA, Voskuhl RR (1999) Estriol ameliorates autoimmune demyelinating disease: implications for multiple sclerosis. Neurology 52: 1230-1238.

28. Weiss L, Or R, Jones RC, Amunugama R, JeBailey L, et al. (2012) Preimplantation factor $\left(\mathrm{PIF}^{*}\right)$ reverses neuroinflammation while promoting neural repair in EAE model. Journal of the neurological sciences 312:146-157.

29. Sicotte NL, Liva SM, Klutch R, Pfeiffer P, Bouvier S, et al. (2002) Treatment of multiple sclerosis with the pregnancy hormone estriol. Ann Neurol 52: 421-428.

30. Voskuhl (2014) A Combination Trial of Estriol Plus Glatiramer Acetate in Relapsing-Remitting Multiple Sclerosis. Annual Meeting of the American Academy of Neurology, Philadelphia, PA, USA.

31. Vukusic S, Ionescu I, El-Etr M, Schumacher M, Baulieu EE et al. (2009) The Prevention of Post-Partum Relapses with Progestin and Estradiol in Multiple Sclerosis (POPART'MUS) trial: rationale, objectives and state of advancement. J Neurol Sci 286: 114-118.

32. Nielsen NM, Jørgensen KT, Stenager E, Jensen A, Pedersen BV, et al. (2011) Reproductive history and risk of multiple sclerosis. Epidemiology 22: 546-552.

33. Hedström AK, Hillert J, Olsson T, Alfredsson L (2014) Reverse causality behind the association between reproductive history and MS. Mult Scler 20: 406-411.

34. Sneed RS, Cohen S, Turner RB, Doyle WJ (2012) Parenthood and host resistance to the common cold. Psychosom Med 74: 567-573.

35. Al-Shammri S, Rawoot P, Azizieh F, AbuQoora A, Hanna M, et al. (2004) Th1/Th2 cytokine patterns and clinical profiles during and after pregnancy in women with multiple sclerosis. J Neurol Sci 222: 21-27.

36. Ostensen M, Villiger PM (2007) The remission of rheumatoid arthritis during pregnancy. Semin Immunopathol 29: 185-191.

37. Stojan G, Baer AN (2012) Flares of systemic lupus erythematosus during pregnancy and the puerperium: prevention, diagnosis and management. Expert Rev Clin Immunol 8: 439-453. 
Citation: Teter B, Kavak KS, Kolb C, Coyle PK, Guttman BW (2014) Parity Associated with Long-Term Disease Progression in Women with Multiple Sclerosis. J Mult Scler 1: 101. doi:10.4172/jmso.1000101

Page 6 of 6

38. Correale J, Arias M, Gilmore W (1998) Steroid Hormone Regulation of Cytokine Secretion by Proteolipid Protein-Specific CD4+ T Cell Clones Isolated from Multiple Sclerosis Patients and Normal Control Subjects. J Immunol 161: 3365-3374.

39. Cutolo M, Sulli A, Straub RH (2014) Estrogen's effects in chronic autoimmune/inflammatory diseases and progression to cancer. Expert Rev Clin Immunol 10: 31-39.

40. Villaggio B, Soldano S, Cutolo M (2012) 1,25-dihydroxyvitamin D3 downregulates aromatase expression and inflammatory cytokines in human macrophages. Clin Exp Rheumatol 30: 934-938.
41. Daumer M, Weinshenker BG, Voskuhl R (2012) Pregnancy: a "modifiable" risk factor in MS? Neurology 78: 846-848.

42. Alwan S, Dybalski M, Yee IM, Greenwood TM, Roger E, et al. (2013) Multiple sclerosis and pregnancy: a comparison study. Can J Neurol Sci 40: 590-596.

43. Ramagopalan S, Yee I, Byrnes J, Guimond C, Ebers G, et al. (2012) Term pregnancies and the clinical characteristics of multiple sclerosis: a population based study. J Neurol Neurosurg Psychiatry 83: 793-795. 